\title{
Subcellular Localization of Disease-Associated Proteins in Psoriasis ${ }^{\dagger}$
}

\author{
D. Subhashini ${ }^{1, *}$, Darapaneni Akhila ${ }^{1}$, Daniel Alex Anand ${ }^{1}$ \\ 1 Department of Bioinformatics and the Centre for Molecular Data Science and Systems Biology, Sathyabama Institute of \\ Science and Technology, Chennai, India \\ * Correspondence: dsubha83@gmail.com; \\ $\dagger$ Presented at International e-Conference on Bioengineering for Health and Environment (ICBHE 2020)
}

Received: 5.07.2020; Revised: 10.07.2020; Accepted: 12.07.2020; Published: 15.07.2020

\begin{abstract}
Psoriasis is an autoimmune, persisting, inflammatory disorder that extremely affects the skin and joints of the system. In spite of the field under investigation across the globe roots toward the origin and the molecular pathophysiology of the disease, yet, the mechanism is vaguely presumed. The pathology has its basis in the underlying genes, the protein interactomes, and the metabolic pathways. Subcellular localization of the proteins (Sl) imparts geometrical details of proteins in a cell. In Sl, Proteins conjoin with suitable proteins to assemble into active complexes in signaling routes and metabolic pathways. Variations in the disease set of genes modify the production of gene outcomes as well alters the choosing steps of appropriate Sl, which interrupts the vital roles of the proteins. Proteins related to the disease are predominantly accumulated in typical Sl, which is why apt recognition of protein Sl guides to track down disease bound proteins and the interdependence between them. To do so, in the current investigation, the GOnet tool has been utilized to identify Sl of the proteins by the input of genes and by modeling and visualizing collaborative graphs in conjunction with GO terms and genes. The results obtained displays that the Psoriasis proteins have been localized in respective cellular compartments such as Golgi apparatus, cytoplasm, nucleolus, mitochondria, peroxisomes cytoskeleton, cytoplasm, endosomes, endoplasmic reticulum, extracellular region, nucleoplasm, cilium, vacuole, protein-containing complex, and nuclear chromosome. Further exploration of subcellular localization followed by protein-protein interaction and molecular pathway analyses may be the bedrock to a deeper insight towards disease development and molecular centered relations alongside multimorbidity interactions in Psoriasis.
\end{abstract}

Keywords: Protein-protein interaction; Subcellular localization; Psoriasis.

(C) 2020 by the authors. This article is an open-access article distributed under the terms and conditions of the Creative Commons Attribution (CC BY) license (https://creativecommons.org/licenses/by/4.0/).

\section{Funding}

This research received no external funding.

\section{Acknowledgments}

This research has no acknowledgment.

\section{Conflicts of Interest}

The authors declare no conflict of interest. 